\title{
Stereoscopic Correspondence of Particles for 3-Dimensional Particle Tracking Velocimetry by using Genetic Algorithm
}

\author{
Sanjeeb Prasad Panday \\ Department of Electronics and Computer Engineering, Pulchowk Campus, Institute of Engineering, \\ Tribhuvan University, Kathmandu, Nepal \\ Corresponding author: sanjeeb@ioe.edu.np
}

Received: July 25, 2016

Revised: Aug. 18, 2016

Accepted: Aug. 25, 2016

\begin{abstract}
The genetic algorithm (GA) based stereo particle-pairing algorithm has been developed and applied to the spatial particle-pairing problem of the stereoscopic three-dimensional (3-D) PTV system. In this 3-D PTV system, particles viewed by two (or more than two) stereoscopic cameras with a parallax have to be correctly paired at every synchronized time step. This is important because the 3-D coordinates of individual particles cannot be computed without the knowledge of the correct stereo correspondence of the particles. In the present study, the GA algorithm is applied to the epipolar line proximity analysis for establishing correspondence of particles pairs between two co-instantaneous stereoscopic particles images, in order to compute the 3-D coordinates of every individual particle. The results are tested with various standard images and it's found that the new strategy using GA works better than conventional particle pairing methods of 3-D particle tracking velocimetry for steoroscopic PTV.
\end{abstract}

Keywords: Particle pairing problem, Genetic algorithm, Particle tracking velocimetry, 3-D PTV, Visualization.

\section{Introduction}

Particle image velocimetry (PIV) has been widely accepted as a reliable whole-field velocity measurement technique in every branch of the fluid engineering [1] and the recent trend goes to the three-dimensional. The main trend of the current 3-D PIV is a stereoscopic extension of a standard 2-D PIV system, using a finite-thickness laser light sheet and two stereoscopic CCD cameras in Scheimpflug optical arrangement [21]. However, much as the feasibility of this type of 3-D PIV is appreciated, the measurement target of this system is limited, by its principle, to 3D flows with relatively small out-of-plane velocity components. If full-volume 3-D flow measurement is required, the 3-D particle tracking velocimetry (PTV) $[6,20]$ is more promising technique than the stereoscopic PIV using Scheimpflug arrangement because the depth velocity component is as well resolved as the other components.

A typical 3-D PTV is composed of two successive steps of particle matching [8]. The first one is the spatial particle pairing, in which the particles viewed by two (or more than two) stereoscopic 
cameras with a parallax (different viewing angles) have to be correctly paired at every synchronized time step. This is quite important because the 3-D coordinates of individual particles cannot be computed without being assured of identification of two particle images. After this step, the second process is the temporal particle pairing, in which the particles with computed 3-D coordinates have to be correctly paired with those at the next time step. Of these two processes of particle pairing, for the temporal particle pairing almost the entire 2-D particle tracking algorithms are applicable without any additional complexity [7, 9, 15, 24]. Two of the present authors have even successfully applied the GA to the temporal particle pairing in their previous article [16]. However, the spatial process of particle pairing always possesses some challenges when 3-D particle coordinates must be calculated with accuracy and with high recovery ratio. The main problem comes from the low velocity recovery ratio with respect to the number of the existing particles. This leads to a low density of measurement velocity vectors that looks much less satisfactory than those of the 2-D particle tracking velocimetry. In this context, for full-volume 3-D flow measurement, the epipolar line nearest-neighbor analysis [10] seems a standard technique of 3-D particle pairing, although there are some variations or modifications (simplified or complicated) reported so far in the literature. However, even within the extent of the standard epipolar line nearest-neighbor analysis, the detail of the algorithm is different from author to author. In most cases, the particle-pairing algorithm is based on the normal distance between a particle centroid in one of the two camera frames and an epipolar line projected on the same camera frame but derived from a particle centroid in the other camera frame. Since this minimization of normal distance in a single camera frame does not necessarily give rise to correct particle pairing, minimization of the sum of the left-camera and right-camera normal distances is often used as a better solution.

Another troublesome factor for the accuracy of particle pairing using the epipolar line nearestneighbor analysis is the existence of candidate particles that are in reality incorrect partners but happen to come in the vicinity of the reference epipolar line. The usual method of avoiding this is to use a third (and sometimes a fourth) stereoscopic camera, which often facilitates the validation of the particle pairing results obtained from the two camera arrangement. However, an additional camera usually complicates the particle pairing strategy furthermore and the whole system requires more powerful, expensive and sophisticated hardware and software. If simple twocamera setup has the possibility of producing more accurate particle pairing results, this will be without doubt the best solution to be adopted in the 3-D PTV.

From such a background, some of the authors have reported some advanced computational techniques for the stereo matching problem of the 3-D PTV. A hopfield neural network based computational strategy [5] was suggested by Grant and Pan [3, 4]. In their approach, the camera configuration is restricted in such a way that the object plane, lens plane and image plane all need to be parallel to each other, and the lens of cameras must be in the same plane. In this kind of translational configuration, there is not enough flexibility to resolve the depth. Moreover, one of the present authors have already reported that the hopfield neural network approach is not a good choice for the particle tracking velocimetry when the particle number exceeds just a small value 
of 150 particles per frame $[11,13]$. Similarly, the neural network based on self-organizing maps (SOM) method [12, 14] and cellular neural network (CNN) method [17] using two-camera arrangement was applied for the stereo PTV. In the case of SOM, the algorithm works well with larger number of particles (say more than 500) but the initialization of the SOM computation parameters is too difficult and in addition, the stereo PTV results are also very sensitive to these computation parameters. On the other hand, the CNN method is indeed an interesting attempt with a concept of the minimization of Lyapunov energy function but in order to get reasonable matching results, the energy function must be composed of four object functions representing the physical constraints of the flow. This often complicates the computation process with an additional problem of the weight balance of the multiple object functions.

With such a view, the present authors have been trying to establish a new particle pairing strategy for stereoscopic particle images obtained from two-camera arrangement. The point of their new strategy was the use of a genetic algorithm (GA) applied to the epipolar line nearest-neighbor analysis. Using this strategy, on one hand, the optimization operation can be carried out as an alternative to conventional methods of epipolar line proximity search, and on the other hand, multiple constraint conditions can be more effectively addressed than the other stereo PTV methods cited above. In the present study, the classical epipolar line nearest-neighbor analysis and similar techniques are improved to some extent by using a genetic strategy aiming at total minimization of normal distances. The accuracy of the spatial particle pairing results is examined by using the PIV Standard Images [18, 19], comprehensive sets of synthetic particle images showing a 3-D turbulent impinging jet.

\section{Genetic Algorithm}

\subsection{Genetic encoding and the initial population}

In the classical stereoscopic PTV, the seeded particles in water or air are visualized and viewed with a volume light source. And then the particles in the left-camera frame image are correctly paired with those in the right-camera image or vice versa. Usually, the centroids of the particles must be extracted in advance through image binarization and labeling processes and the particles are assigned their own unique labels (ID numbers). This first step, rather important in the case of real particle images from visualization experiments, is not necessary in the present PIV Standard Images, because the particle centroid data come with the relevant synthetic images. The second step then is the GA encoding of the physical problem in the form of a genetic population composed of specific chromosomes. In the case of the simple genetic algorithm (SGA), the gene codes are given with simple binary bits, while in many applications of optimization problems (e.g. a traveling salesman's problem), they are often given by integer numbers representing some specific variables of the physical problem. In the present genetic algorithm, the encoding scheme as shown in Fig. 1 has been used for the spatial (stereoscopic) matching. Every individual of the population consists of two integer-numbered chromosomes (or gene strings) and each chromosome gene string represents a sequence of left-frame and right-frame particle IDs (or between two subsequent image pairs), and numbers which are at the same gene position between 
two chromosomes indicate the corresponding particle between right and left frames (or between two time-differential image frames). The goal of the algorithm is to create a best-fit biological individual with respect to the ideal environment needed by the constraints of the physical problems and thus to approach gradually to the most probable particle match result.

To be more precise, one of the two chromosomes of individuals at the first generation is represented by a string of serial numbers starting from 1, while the other one by randomly ordered serial numbers without any duplication. The number of individuals is fixed all through the computation. The first generation individuals, after their evolution, get encountered with an iterative process of genetic operations consisting of fitness estimation, selection, reproduction, crossover, and mutation. Meanwhile, the two chromosomes of these individuals gradually give rise to better-fit pairs of gene codes and at the final generation, two chromosomes of the best-fit individual are expected to indicate the correct matches of particle ID numbers.

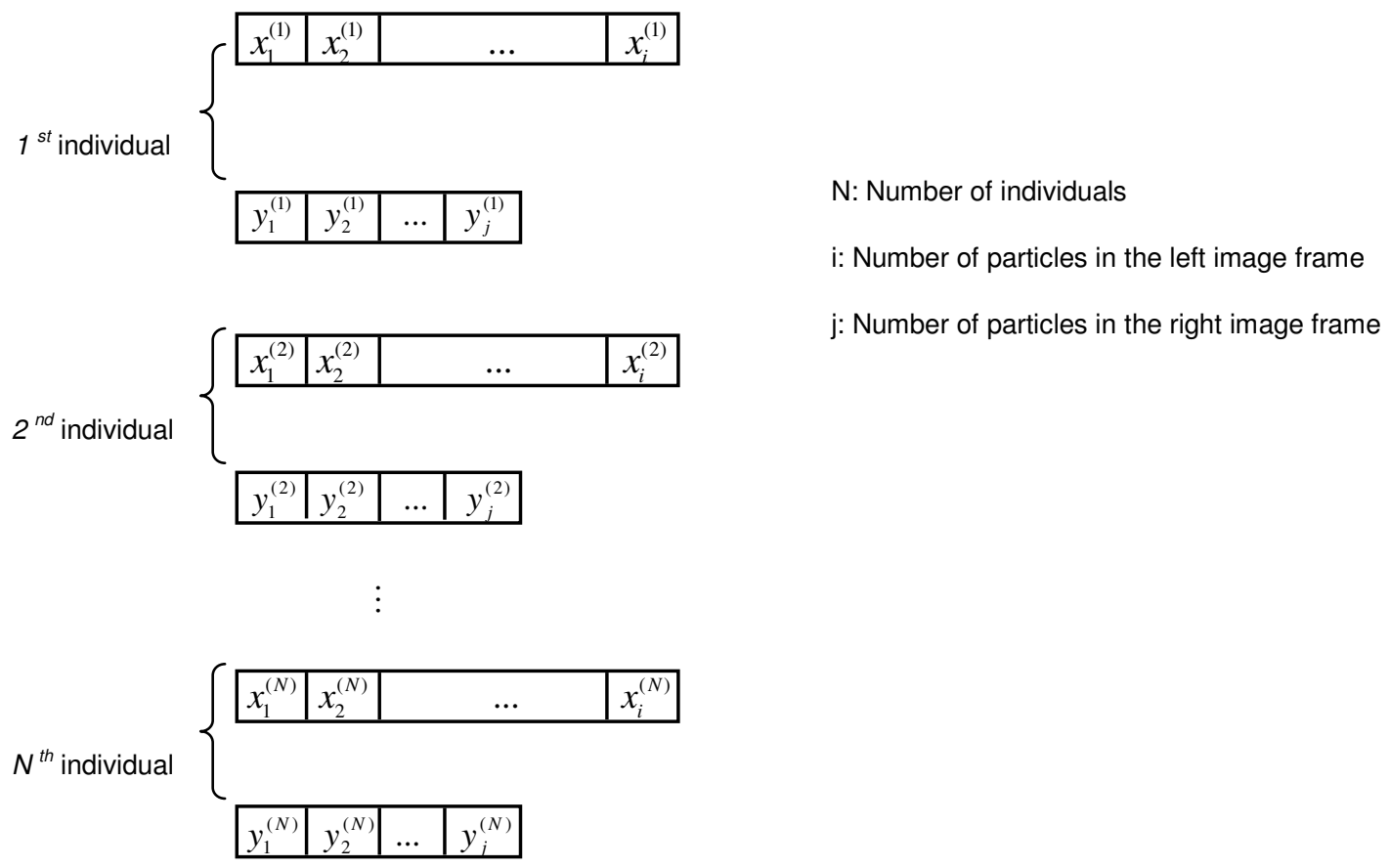

Fig. 1: Genetic encoding scheme used for the stereoscopic particle matching

\subsection{Fitness estimation of individuals}

The fitness of the individuals is estimated by several kinds of 2-D and 3-D distance parameters based on the geometry of particle centroids and the relevant epipolar lines.

\subsubsection{Epipolar lines}

The only physical criterion, which is available for establishment of the stereo correspondence of the particles, is the geometric constraint of the epipolar line. As explained earlier, it has been the 
important part of the fitness (object) function, and thus the major basis for the GA based particle pairing. Knowing the orientation parameters of the cameras from the calibration procedure and proceeding from a particle position, say $\left(X_{1}, Y_{1}\right)$ in one image, an epipolar line in another image can be calculated, on which the corresponding point has to be found. The mathematical expression for 2-D and 3-D epipolar lines, shown in Fig. 2, can be all formulated from the mapping equations (1)-(4), representing the perspective transform [22].

$$
\begin{aligned}
& X_{1}=a_{11} x+a_{12} y+a_{13} z+a_{14}-a_{31} x X_{1}-a_{32} y X_{1}-a_{33} z X_{1} \\
& Y_{1}=a_{21} x+a_{22} y+a_{23} z+a_{24}-a_{31} x Y_{1}-a_{32} y Y_{1}-a_{33} z Y_{1} \\
& X_{2}=b_{11} x+b_{12} y+b_{13} z+b_{14}-b_{31} x X_{2}-b_{32} y X_{2}-b_{33} z X_{2} \\
& Y_{2}=b_{21} x+b_{22} y+b_{23} z+b_{24}-b_{31} x Y_{2}-b_{32} y Y_{2}-b_{33} z Y_{2}
\end{aligned}
$$

where $x, y$ and $z$ are the physical-space 3-D coordinates of a particle centroid, $X_{1}$ and $Y_{1}$ are the 2D projection coordinates on the left-camera screen, whereas $X_{2}$ and $Y_{2}$ are those on the right camera screen. The two sets of matrix coefficients $a_{x x}$ and $b_{x x}$ are respectively the left and right camera parameters, determined by a calibration process using a certain number of calibrated target points viewed by the same two cameras. Equations (1)-(4) can be rearranged and written as shown in equations (5)-(8) respectively.

$$
\begin{aligned}
& X_{1}-a_{14}=\left(a_{11}-a_{31} X_{1}\right) x+\left(a_{12}-a_{32} X_{1}\right) y+\left(a_{13}-a_{33} X_{1}\right) z \\
& Y_{1}-a_{24}=\left(a_{21}-a_{31} Y_{1}\right) x+\left(a_{22}-a_{32} Y_{1}\right) y+\left(a_{23}-a_{33} Y_{1}\right) z \\
& X_{2}-b_{14}=\left(b_{11}-b_{31} X_{2}\right) x+\left(b_{12}-b_{32} X_{2}\right) y+\left(b_{13}-b_{33} X_{2}\right) z \\
& Y_{2}-b_{24}=\left(b_{21}-b_{31} Y_{2}\right) x+\left(b_{22}-b_{32} Y_{2}\right) y+\left(b_{23}-b_{33} Y_{2}\right) z
\end{aligned}
$$

Above equations (5)-(8) can be written in short forms as shown in equations (9)-(12) respectively.

$$
\begin{aligned}
& W_{1} x+W_{2} y+W_{3} z=W_{4} \\
& W_{5} x+W_{6} y+W_{7} z=W_{8} \\
& W_{9} x+W_{10} y+W_{11} z=W_{12} \\
& W_{13} x+W_{14} y+W_{15} z=W_{16}
\end{aligned}
$$

where $W_{1}=a_{11}-a_{31} X_{1}, W_{2}=a_{12}-a_{32} X_{1}, W_{3}=a_{13}-a_{33} X_{1}$ and $W_{4}=X_{1}-a_{14}$. Similarly, it is easy to understand the equivalence of variables $W_{5}$ to $W_{16}$ in reference to equations (6)-(8) and (10)-(12). Now, equations (9) and (10) can be expressed as:

$$
\begin{gathered}
y=\frac{W_{7} W_{1}-W_{3} W_{5}}{W_{2} W_{5}-W_{1} W_{6}} z+\frac{W_{4} W_{5}-W_{1} W_{8}}{W_{2} W_{5}-W_{1} W_{6}} \\
x=\frac{W_{7} W_{2}-W_{6} W_{3}}{W_{1} W_{6}-W_{2} W_{5}} z+\frac{W_{4} W_{6}-W_{2} W_{8}}{W_{1} W_{6}-W_{2} W_{5}}
\end{gathered}
$$

Let, 


$$
\begin{array}{ll}
\frac{W_{7} W_{1}-W_{3} W_{5}}{W_{2} W_{5}-W_{1} W_{6}}=W_{17}, & \frac{W_{4} W_{5}-W_{1} W_{8}}{W_{2} W_{5}-W_{1} W_{6}}=W_{18}, \\
\frac{W_{7} W_{2}-W_{6} W_{3}}{W_{1} W_{6}-W_{2} W_{5}}=W_{19}, & \frac{W_{4} W_{6}-W_{2} W_{8}}{W_{1} W_{6}-W_{2} W_{5}}=W_{20}
\end{array}
$$

Substituting, in equations (13) and (14), we get:

$$
\begin{aligned}
& y=W_{17} z+W_{18} \\
& x=W_{19} z+W_{20}
\end{aligned}
$$

Substituting the value of $x$ and $y$ in equations (15) and (16) to equations (11) and (12), we get:

$$
\begin{aligned}
& \left(W_{9} W_{19}+W_{10} W_{17}+W_{11}\right) z=W_{12}-W_{9} W_{20}-W_{10} W_{18} \\
& \left(W_{13} W_{19}+W_{14} W_{17}+W_{15}\right) z=W_{16}-W_{13} W_{20}-W_{14} W_{18}
\end{aligned}
$$

Equating and cross-multiplication of equations (17) and (18) yields:

$\left(W_{12}-W_{9} W_{20}-W_{10} W_{18}\right)\left(W_{13} W_{19}+W_{14} W_{17}+W_{15}\right)=\left(W_{16}-W_{13} W_{20}-W_{14} W_{18}\right)\left(W_{9} W_{19}+W_{10} W_{17}+W_{11}\right)$

Now, from equations (7), (8), (11), (12) and (19), we get:

$\left(P_{L} X_{2}-Q_{L}\right)\left(-R_{L} Y_{2}+S_{L}\right)=\left(P_{L} Y_{2}-T_{L}\right)\left(-R_{L} X_{2}+U_{L}\right)$

where,

$P_{L}=\left(1+b_{31} W_{20}+b_{32} W_{18}\right)$

$Q_{L}=\left(b_{14}+b_{11} W_{20}+b_{12} W_{18}\right)$

$R_{L}=\left(b_{31} W_{19}+b_{32} W_{17}+b_{33}\right)$

$S_{L}=\left(b_{21} W_{19}+b_{22} W_{17}+b_{23}\right)$

$T_{L}=\left(b_{24}+b_{21} W_{20}+b_{22} W_{18}\right)$ and

$U_{L}=\left(b_{11} W_{19}+b_{12} W_{17}+b_{13}\right)$

Rearranging equation (20), we get:

$P_{i} X_{2}+Q_{i} Y_{2}+R_{i}=0$

where $P_{i}=\left(P_{L} S_{L}-T_{L} R_{L}\right), \quad Q_{i}=\left(-P_{L} U_{L}+Q_{L} R_{L}\right)$ and $R_{i}=\left(T_{L} U_{L}-Q_{L} S_{L}\right)$.

Equation (21) is the epipolar line, in the right frame, corresponding to the particle in the position $\left(X_{1}, Y_{l}\right)$ of the left frame. To generalize the situation, $\left(X_{1}, Y_{l}\right)$ is replaced by $\left(X_{i}, Y_{i}\right)$ in the above computations. Now, the normal distance of the epipolar line in right frame from the position $\left(X_{k}\right.$, $Y_{k}$ ), also in right frame, is given by the equation (22).

$$
d_{i k}=\frac{\left|P_{i} X_{k}+Q_{i} Y_{k}+R_{i}\right|}{\sqrt{P_{i}^{2}+Q_{i}^{2}}}
$$

The value of $d_{i k}$, thus obtained, becomes a part of equation (23) (Section 2.2.2 Fitness function) for each $k^{\text {th }}$ particle in the right frame from the epipolar line drawn in reference of each $i^{\text {th }}$ particle of the left frame. The process goes similarly for calculating $d_{k i}$ for each $i^{\text {th }}$ particle in the left frame from the epipolar line drawn in reference of each $k^{\text {th }}$ particle of the right frame. 


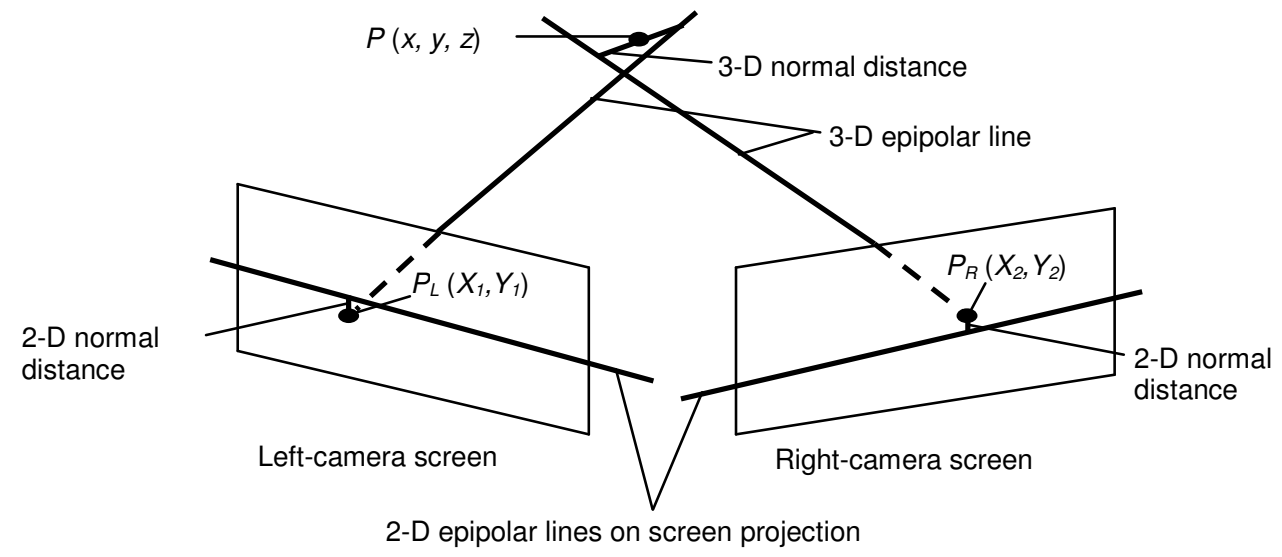

Fig. 2: Epipolar lines (2-D and 3-D)

\subsubsection{Fitness functions}

In equations (1) - (4) in section 2.2.1, if either set of $\left(X_{1}, Y_{1}\right)$ or $\left(X_{2}, Y_{2}\right)$ is given, the other set of $X$ and $Y$ comes into a linear relation, providing an arithmetic equation of a 2-D epipolar line. And if the set of $\left(X_{1}, Y_{1}\right)$ is given in the equations (1) and (2), then the coordinates $x, y$ and $z$ are interrelated as a 3-D epipolar line equation. So are the set of $\left(X_{2}, Y_{2}\right)$ and the equations (3) and (4). Bearing these 2-D and 3-D epipolar distances in mind, the authors have tried in the present experiment four types of fitness functions. The first one given by equation (23) is defined as the total sum of 2-D normal distances between a reference particle and its pairing epipolar line in the same 2-D projection image. For more accuracy, this distance parameter is re-evaluated by addition of another 2-D epipolar line distance obtained from a backtracking pairing process, namely the distance between the relevant respective particle centroid and the epipolar line in the other projection image. To illustrate mathematically, let $E_{i}(i=1,2,3 \ldots N)$ be the epipolar lines in the right frame corresponding to particles $P_{i}(i=1,2,3 \ldots N)$ in the left frame and $E_{k}(k=1,2$, $3 \ldots N)$ be the epipolar lines in the left frame corresponding to particles $P_{k}(k=1,2,3 \ldots N)$ in the right frame. Let, $d_{i k}$ be the distance of the particle $P_{k}$ from $E_{i}$, and the $d_{k i}$ likewise. Now, the genetic strategy is aimed for the minimization of the fitness function: $F=\left(d_{i k}+d_{k i}\right)$. The second one given by equation (24) is defined as the total sum of the 3-D normal distances between two 3-D epipolar lines of pairing particles. The third one given by equation (25) is the total sum of the square of the 2-D normal distances in the same re-evaluation scheme as in equation (23) and the fourth one given by equation (26) is the total sum of the square of the 3-D normal distances. However, as a result of a series of trial computations, the third and fourth fitness definitions, i.e., equations (25) and (26) have not demonstrated any noticeable improvement of pairing results if compared to the first and the second ones. So in the following sections, the genetic algorithm for particle pairing uses only the first and second fitness functions, i.e., equations (23) and (24).

$$
\begin{aligned}
& F F_{1}=\sum_{i}\left(E P L_{i}+E P R_{i}\right)=d_{i k}+d_{k i} \\
& F F_{2}=\sum_{i}\left(E P T_{i}\right)
\end{aligned}
$$




$$
\begin{aligned}
& F F_{3}=\sum_{i}\left(E P L_{i}+E P R_{i}\right)^{2} \\
& F F_{4}=\sum_{i}\left(E P T_{i}\right)^{2}
\end{aligned}
$$

\subsection{Genetic Operations}

After the fitness estimation of individuals, the first operation is the selection and reproduction of better-fit individuals. As a general principle of the genetic algorithm, a better-fit individual must have more probability of being reproduced in the following generation. This is a basic rule of selection and reproduction. The next genetic operation is crossover of randomly selected two individuals. Either of the two gene strings picked up from two randomly selected individuals is crossed over at the same randomly determined string position. This operation provides the first opportunity for the individuals to have new combinations of particle ID's between the left- and right-camera frames. And finally, the last operation is mutation, in which two randomly selected gene codes in a single chromosome of a randomly selected individual are exchanged at a prescribed rate. This is the second chance for the individuals to have new combinations of particle ID's. Unlike most of the conventional genetic operations, the present algorithm uses some new ideas that are introduced in addition to the basic genetic operations with a view to accelerate the computation.

Relating to the genetic encoding scheme shown in Fig.1, the selection and reproduction are the operations done on the individual basis without changing the combinations of particle IDs in the two gene strings. But the crossover and mutation are operated in only one of the two gene strings and, therefore, sure to change the combinations of particle IDs.

\subsubsection{Selection and reproduction}

The present study employs the ranking selection (more precisely, the linear or exponential ranking selection) method. In addition, the present method adopts a widely-used option known as an elitist preserving selection, in which the best-fit ever-generated individual is unconditionally reproduced in the next generation.

\subsubsection{Sort of gene strings}

This one, not included in the conventional genetic operations, is a unique aspect of the present genetic algorithm. At each generation, the two gene strings in the best-fit individual (or more than two best-fit individuals) are sorted simultaneously according to the fitness index of each gene pair. This simultaneous sort operation retains any combination of particle IDs between the first and second gene strings.

\subsubsection{Crossover}

Crossover is always a unique opportunity in which two gene strings coming from either one of the two chromosomes in two randomly-selected individuals is crossed over with each other. In the present study, a single point partially matched crossover method as shown in Fig. 3 is used. 
18 Stereoscopic Correspondence of Particles for 3-Dimensional Particle Tracking Velocimetry by using...

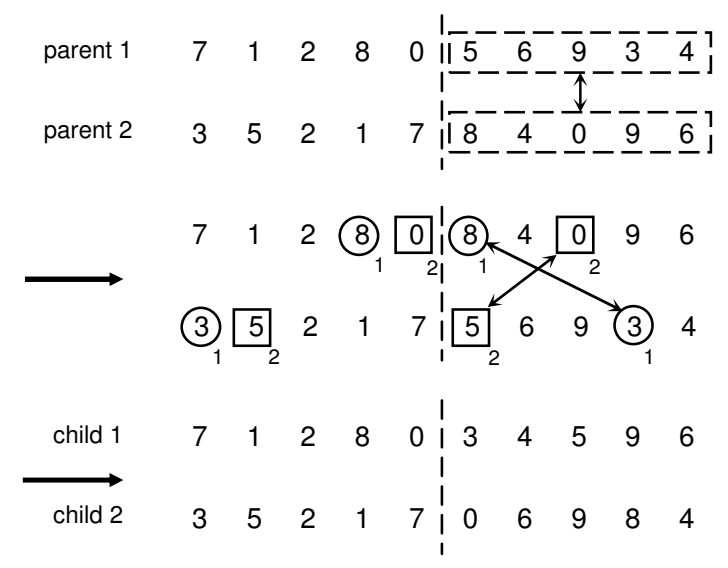

Fig. 3: Partially matched crossover (Single-Point)

\subsubsection{Mutation}

In this mutation operation, two randomly selected gene codes in either of the two gene strings are exchanged in a given number of randomly selected individuals. This is depicted clearly in Fig. 4.

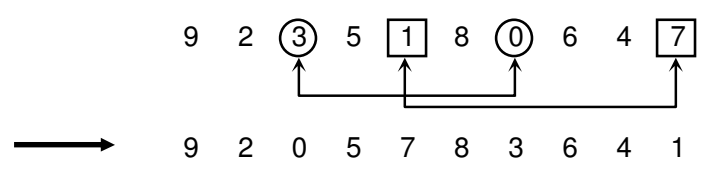

Fig. 4: Mutation based on random gene-exchange

\section{Camera Calibration}

The sets $a_{x x}$ and $b_{x x}$ in equations (1)-(4) are the camera parameters for the left and right camera and are the essential components to determine the equation of the epipolar line as mentioned above. These parameters measure the position and orientation of the camera with respect to a world coordinate system. The solution to determine these parameters is formulated as shown in equation (27). This representation is same as the representations made in equations (1)-(2). This is the case for the determination of the camera parameters of the left camera. Process goes similar to the computations of the right camera parameters too. An over determined set of linear equations is obtained by configuring equation (27) for each calibration target. Minimum numbers of 6 calibration targets are required to solve the 11 unknown calibration parameters. Suppose equation (27) is expressed as in the equation (28) form replacing the components from left to right by $J_{c}, M_{c}$, and $N_{c}$ respectively then equation (28) can be solved for calibration parameter matrix $N_{c}$ by using linear least-squares technique as the pseudo-inverse [2, 23] as in equation (29). 


$$
\left(\begin{array}{l}
X \\
Y
\end{array}\right)=\left(\begin{array}{cc}
x & 0 \\
y & 0 \\
z & 0 \\
1 & 0 \\
0 & x \\
0 & y \\
0 & z \\
0 & 1 \\
-x X & -x Y \\
-y X & -y Y \\
-z X & -z Y
\end{array}\right)^{T}\left(\begin{array}{l}
a_{11} \\
a_{12} \\
a_{13} \\
a_{14} \\
a_{21} \\
a_{22} \\
a_{23} \\
a_{24} \\
a_{31} \\
a_{32} \\
a_{33}
\end{array}\right)
$$

$J_{c}=M_{c} N_{c}$

$N_{c}=\left(M_{c}{ }^{T} M_{c}\right)^{-1} M_{c}{ }^{T} J_{c}$

\section{Experimental Results}

The GA particle-matching algorithm is tested by using the 3-D PIV Standard Images [18, 19] available from the Visualization Society of Japan. These images are composed of various sets of synthetic time-series particle images generated from DNS (Direct Numerical Simulation) results of a 3-D impinging jet in a square cavity and 6 sets of them are stereoscopic images for with different portions of the flow field and/or different viewing angles of the cameras. One of the advantages of these standard images is that the datasets come with text files of the original particle coordinates, so that one can compare the analysis results of particle matching with the correct data. In the present study, the matching results are presented for four stereoscopic particles image sets namely \#351, \#352, \#371 and \#377 out of six, because these four are especially suitable for basic testing of every 3-D PIV or PTV system. Sample frames for each of these image series are shown in Fig. 5 and Table 1 gives the summary of specifications of these Standard Images $[18,19]$. The first two images sets (\#351 and \#352) represent the same portion of the flow field with same viewing angles of the cameras. The only difference between the two is the number of particles distributed in the view field. For the first three series (\#351, \#352 and $\# 371$ ), the left and right (stereoscopic) cameras are fixed symmetrically with their optical axes in plane. The symmetry comes from the incidence angle of $\pm 30^{\circ}$ of the two camera axes with respect to the $x$-axis. In the first two series (\#351 and \#352), the plane of the two camera axes is parallel to the $x-y$ plane while in the third one (\#371), the camera axes have also a yaw angles of $-10^{\circ}$ with respect to the $x-y$ plane. For the \#377 series, the cameras are in more complex optical arrangement as specified in Table 1 . In any of the four series, in order to simulate particle refraction effects in really experimental environments, the use of cylindrical volume illumination and water refractive index of 1.33 are taken into account.

For determining camera parameters, pair of (left and right) calibration images is provided for these series of particle images. In case of image series \#351, \#352 and \#377, there are 27 
stationary marker particles whereas in the case of image series \#371, there are 125 stationary marker particles. The marker particles used for the calibration purposes are shown in Fig. 6. As \#351 and \#352 image series correspond to the same optical conditions, the calibration marker particles are common for both of these series.

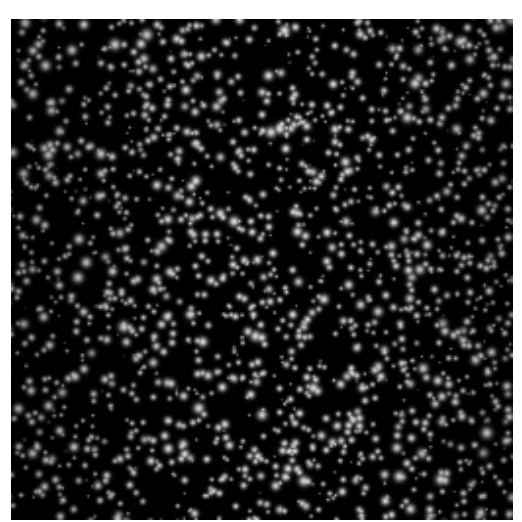

(a) Left frame \#351

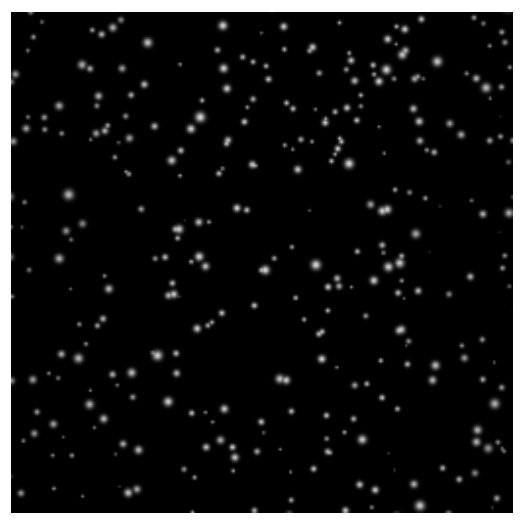

(c) Left frame \#352

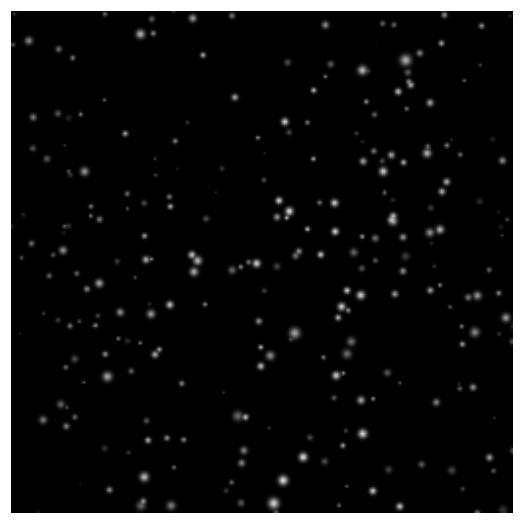

(e) Left frame \#371

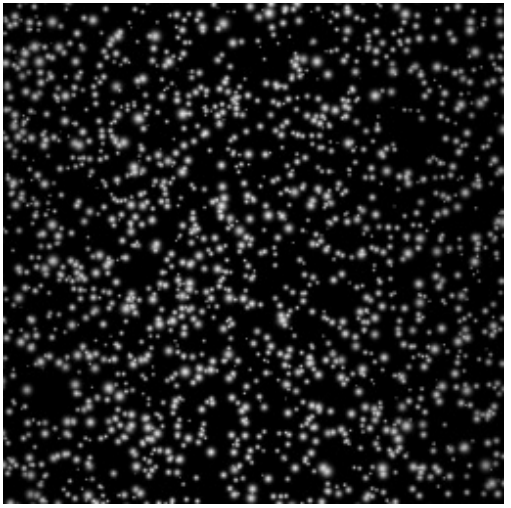

(b) Right frame \#351

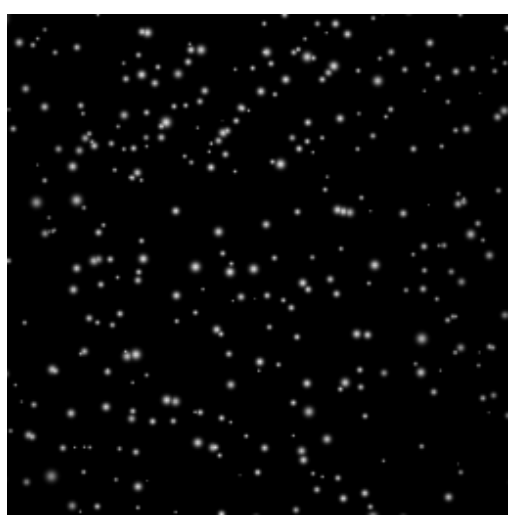

(d) Right frame \#352

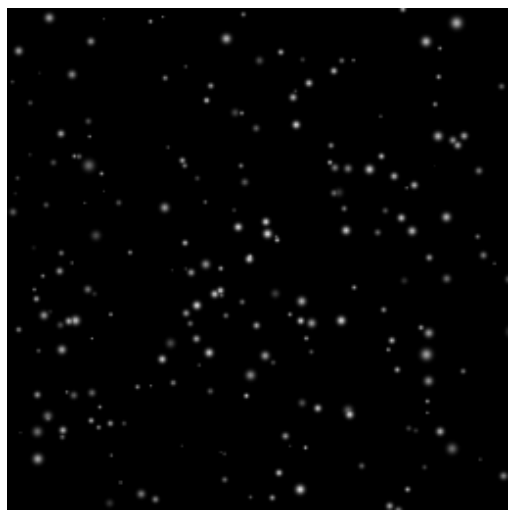

(f) Right frame \#371 


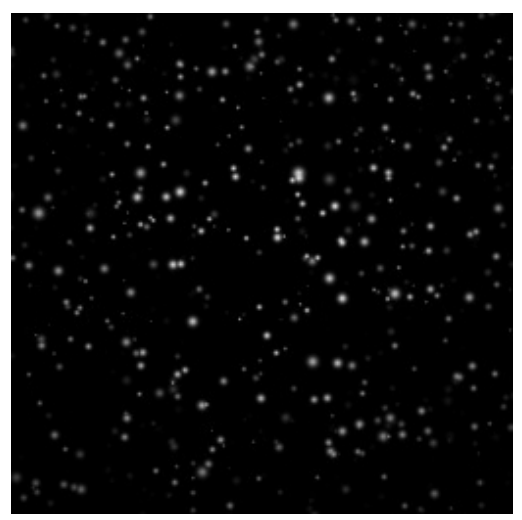

(g) Left frame \#377

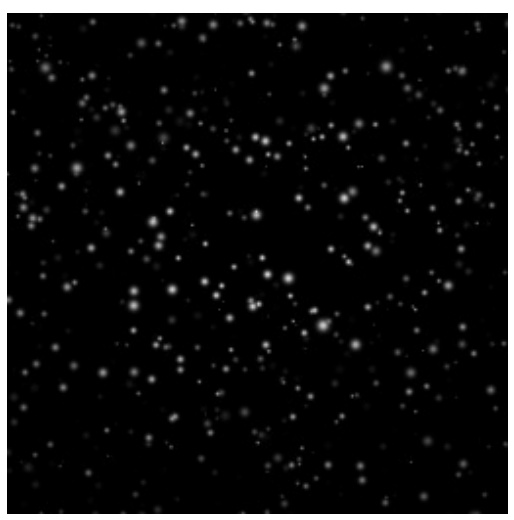

(h) Right frame \#377

Fig. 5: Standard images (Left and Right frames)

Table 1 : Summary of different 3-D PIV Standard Images

\begin{tabular}{|c|c|c|c|c|c|}
\hline \multicolumn{2}{|c|}{ Series \# / Frame \# } & $352 / 0000$ & $351 / 0000$ & $371 / 0000$ & $377 / 0000$ \\
\hline \multicolumn{2}{|c|}{ Number of existing particles } & 372 & 2092 & 366 & 939 \\
\hline \multicolumn{2}{|c|}{ Mean particle diameter } & 5 pix & 5 pix & 5 pix & 5 pix \\
\hline \multicolumn{2}{|c|}{ Standard deviation of diameter } & 2 pix & 2 pix & 2 pix & 2 pix \\
\hline \multicolumn{2}{|c|}{ Minimum particle diameter } & 1 pix & 1 pix & 1 pix & 1 pix \\
\hline \multicolumn{2}{|c|}{ Volume of visualized flow (in situ) } & $2 \mathrm{~cm}^{3}$ & $2 \mathrm{~cm}^{3}$ & $1 \mathrm{~cm}^{3}$ & $0.5 \mathrm{~cm}^{3}$ \\
\hline \multicolumn{2}{|c|}{ Maximum flow rate (in situ) } & $12 \mathrm{~cm} / \mathrm{sec}$ & $12 \mathrm{~cm} / \mathrm{sec}$ & $12 \mathrm{~cm} / \mathrm{sec}$ & $12 \mathrm{~cm} / \mathrm{sec}$ \\
\hline \multicolumn{2}{|c|}{ Refraction index } & 1.33 & 1.33 & 1.33 & 1.33 \\
\hline \multicolumn{2}{|c|}{ Number of calibr. marker particles } & 27 & 27 & 125 & 27 \\
\hline \multirow{4}{*}{$\begin{array}{l}\text { Left } \\
\text { camera }\end{array}$} & Distance to origin center & $20 \mathrm{~cm}$ & $20 \mathrm{~cm}$ & $20 \mathrm{~cm}$ & $11.5 \mathrm{~cm}$ \\
\hline & Inclination from $\mathrm{x}$-axis & $-30 \mathrm{deg}$ & $-30 \mathrm{deg}$ & $-30 \mathrm{deg}$ & $-29.9 \mathrm{deg}$ \\
\hline & Inclination from $y$-axis & $0 \mathrm{deg}$ & $0 \mathrm{deg}$ & $-10 \mathrm{deg}$ & $-45 \mathrm{deg}$ \\
\hline & Inclination from $\mathrm{z}$-axis & $0 \mathrm{deg}$ & $0 \mathrm{deg}$ & $0 \mathrm{deg}$ & $16.1 \mathrm{deg}$ \\
\hline \multirow{4}{*}{$\begin{array}{l}\text { Right } \\
\text { camera }\end{array}$} & Distance to origin center & $20 \mathrm{~cm}$ & $20 \mathrm{~cm}$ & $20 \mathrm{~cm}$ & $11.5 \mathrm{~cm}$ \\
\hline & Inclination from $\mathrm{x}$-axis & $30 \mathrm{deg}$ & $30 \mathrm{deg}$ & $30 \mathrm{deg}$ & $0 \mathrm{deg}$ \\
\hline & Inclination from y-axis & $0 \mathrm{deg}$ & $0 \mathrm{deg}$ & $-10 \mathrm{deg}$ & $-90 \mathrm{deg}$ \\
\hline & Inclination from $\mathrm{z}$-axis & $0 \mathrm{deg}$ & $0 \mathrm{deg}$ & $0 \mathrm{deg}$ & $30 \mathrm{deg}$ \\
\hline
\end{tabular}




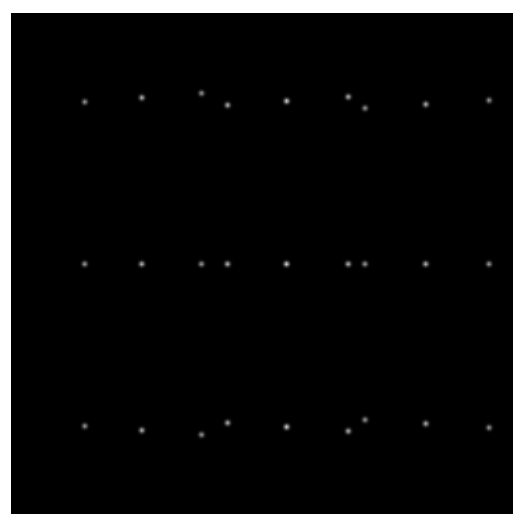

(a) Left frame \#351, \#352

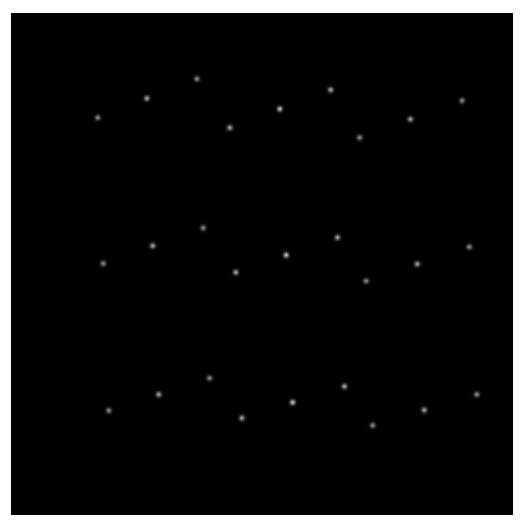

(c) Left frame \#371

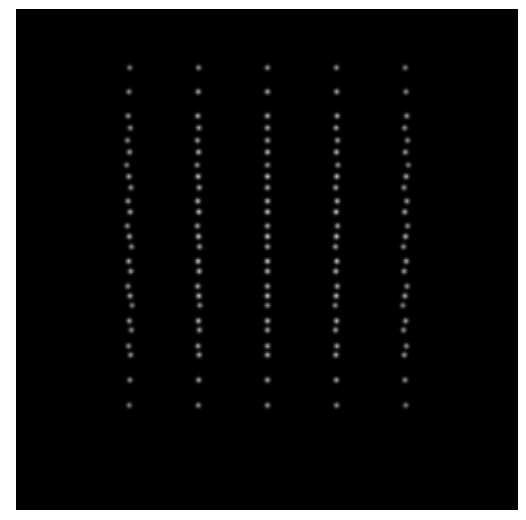

(e) Left frame \#377

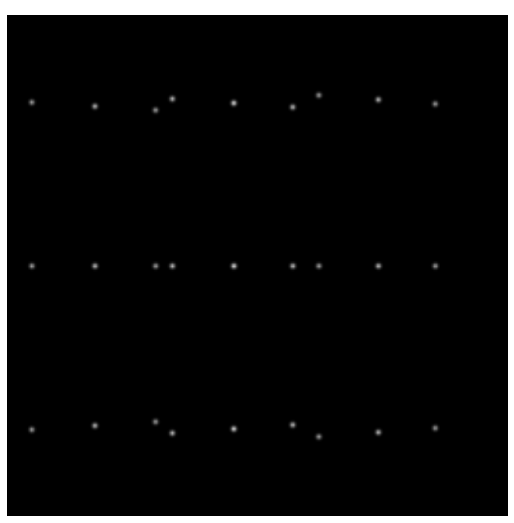

(b) Right frame \#351, \#352

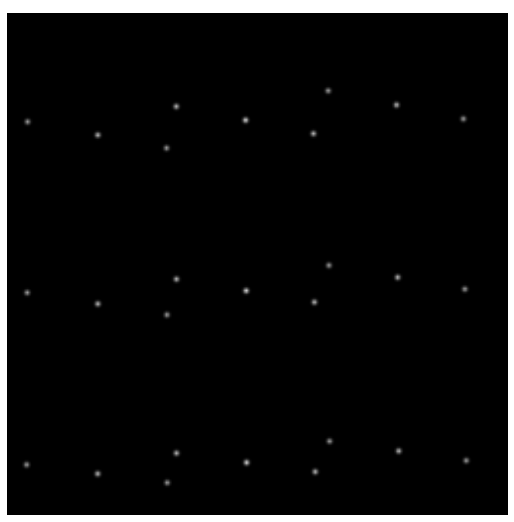

(d) Right frame \#371

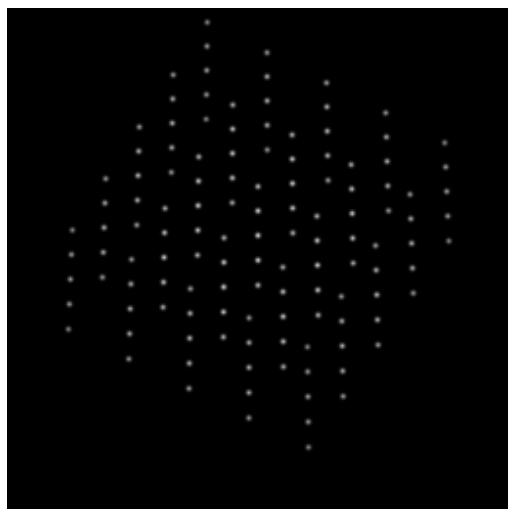

(f) Right frame \#377

Fig. 6: Left and Right image frames for calibration

The stereoscopic particle matching results using the particle coordinates data of these sets of images are summarized in Table 2. This table includes the results for both varied seeding densities as well as the different optical aspects of the stereoscopic particle velocimetry with or without incidence, yaw, and roll angles of the cameras as detailed in Table 1. The abbreviation 
$\mathrm{p} / \mathrm{p}$ in this table stands for "dataset with only perfect pairs", which is generated with an additional data processing by discarding all the loss-of-pair particles. By contrast, the datasets without abbreviation $\mathrm{p} / \mathrm{p}$ include a certain degree of loss-of-pair particles that were present in the original data. Two different numbers of particles for this type of datasets in Table 2 reflects this background. The "correct pair rate" in both these cases means the ratio of total number of correctly matched particles to the total number of matchable particles. The results obtained with conventional method of pairing, i.e., without using GA are also presented side by side in Table 2 . The results obtained with conventional method of pairing (without using GA) are also presented side by side in Table 2 . This results using conventional method (particle pairing without GA) come from particle matching using a conventional epipolar-line proximity analysis based on Nishino et al. [10] where the particles $i$ and $k$ which are able to produce the minimum value of $d_{i k}$ $+d_{k i}$ are considered as perfect pairs.

Table 2 : Results of stereoscopic particle pairing

\begin{tabular}{|c|c|c|c|c|c|c|}
\hline \multirow[b]{2}{*}{$\begin{array}{l}\text { Series \# / } \\
\text { Frame \# }\end{array}$} & \multicolumn{3}{|c|}{$\begin{array}{l}\text { Particle pairing without GA } \\
\text { (Conventional method) }\end{array}$} & \multicolumn{3}{|c|}{ Particle pairing with GA } \\
\hline & $\begin{array}{l}\text { Number of } \\
\text { existing particle } \\
\text { pairs }\end{array}$ & $\begin{array}{l}\text { Number of } \\
\text { correct } \\
\text { pairs }\end{array}$ & $\begin{array}{l}\text { Correct } \\
\text { pair rate }\end{array}$ & $\begin{array}{l}\text { Number of } \\
\text { existing particle } \\
\text { pairs }\end{array}$ & $\begin{array}{l}\text { Number of } \\
\text { correct } \\
\text { pairs }\end{array}$ & $\begin{array}{l}\text { Correct } \\
\text { pair rate }\end{array}$ \\
\hline$\# 351 / 000$ & $1546(\mathrm{p} / \mathrm{p})$ & 1253 & $81.04 \%$ & $1546(\mathrm{p} / \mathrm{p})$ & 1317 & $85.19 \%$ \\
\hline$\# 351 / 000$ & (1817 / 1818) & 1224 & $79.17 \%$ & $(1817 / 1818)$ & 1236 & $79.94 \%$ \\
\hline \#351/001 & $1526(\mathrm{p} / \mathrm{p})$ & 1249 & $81.84 \%$ & $1526(\mathrm{p} / \mathrm{p})$ & 1298 & $85.05 \%$ \\
\hline \#351/001 & $(1801 / 1802)$ & 1228 & $80.47 \%$ & $(1801 / 1802)$ & 1239 & $81.19 \%$ \\
\hline \#352/000 & $283(\mathrm{p} / \mathrm{p})$ & 266 & $93.99 \%$ & $283(\mathrm{p} / \mathrm{p})$ & 275 & $97.17 \%$ \\
\hline$\# 352 / 000$ & $(325 / 328)$ & 264 & $93.28 \%$ & $(325 / 328)$ & 267 & $94.35 \%$ \\
\hline \#352/001 & $277(\mathrm{p} / \mathrm{p})$ & 259 & $92.83 \%$ & $277(\mathrm{p} / \mathrm{p})$ & 269 & $97.11 \%$ \\
\hline \#352/001 & $(323$ / 329) & 257 & $92.11 \%$ & $(323 / 329)$ & 263 & $94.26 \%$ \\
\hline \#371/000 & $157(\mathrm{p} / \mathrm{p})$ & 141 & $89.80 \%$ & $157(\mathrm{p} / \mathrm{p})$ & 147 & $93.63 \%$ \\
\hline \#371/000 & $(267 / 214)$ & 138 & $87.89 \%$ & $(267 / 214)$ & 140 & $89.17 \%$ \\
\hline \#371/001 & $160(\mathrm{p} / \mathrm{p})$ & 145 & $90.62 \%$ & $160(\mathrm{p} / \mathrm{p})$ & 152 & $95.0 \%$ \\
\hline$\# 371 / 001$ & $(265 / 218)$ & 141 & $88.12 \%$ & $(265 / 218)$ & 145 & $90.63 \%$ \\
\hline \#377/000 & $352(\mathrm{p} / \mathrm{p})$ & 265 & $75.28 \%$ & $352(\mathrm{p} / \mathrm{p})$ & 279 & $79.26 \%$ \\
\hline \#377/000 & $(571 / 563)$ & 238 & $67.61 \%$ & $(571 / 563)$ & 255 & $72.44 \%$ \\
\hline \#377/001 & $352(\mathrm{p} / \mathrm{p})$ & 269 & $76.42 \%$ & $352(\mathrm{p} / \mathrm{p})$ & 282 & $80.11 \%$ \\
\hline \#377/001 & $(573$ / 569) & 248 & $70.45 \%$ & $(573 / 569)$ & 258 & $73.29 \%$ \\
\hline
\end{tabular}

The values of the important GA parameters used for the stereoscopic particle matching are as follows: 
Number of individuals = 5 (for Image Series \# 352, \#371 and \# 377) and 10 (for Image Series \# 351)

Number of iterations = 5000 (for Image Series \# 352, \#371 and \# 377) and 10000 (for Image Series \# 351)

Selection rate $=0.2$

Crossover rate $=0.2$

Mutation rate $=0.05$

From Table 2, it is recognized that the correct pair rate is more or less increased with the use of the genetic algorithm, regardless of which series of image datasets and frames are used. In the case of \#351, the number density of particles is almost 6 times as much as \#352 but nonetheless, the correct pair rate amounts to $85 \%$ for the perfect-pair particle dataset. This indicates that the genetic algorithm particle matching works rather stably even in application to high-density particle images and this is also an indicative of an increased effectiveness of the GA-based stereoscopic particle pairing for rather densely seeded particle images. The maximal pair rate of $97.0 \%$ (perfect-pair particle dataset of \#352) may be considered as a limit of the stereoscopic particle matching using two-camera arrangement and for more accuracy, assistance by the third and/or fourth camera will be needed. Finally, Fig. 7 shows the comparison between the 3-D locations of the detected particles using the current GA algorithm and the correct 3-D locations of the particles, which is obtained from the original data sets. This figure explicitly shows the effectiveness as well as the accuracy of the current GA algorithm.

Another merit of this GA approach is that the performance is considerably stable regardless of the optical conditions of particle imaging. Even with or without incidence, yaw and roll angles of the two stereoscopic cameras, the correct pairing results are maintained at a constantly high level. This is certainly important when the stereoscopic PTV has to be employed in many industrial applications, where the positions of cameras and of laser light units are more or less restricted.

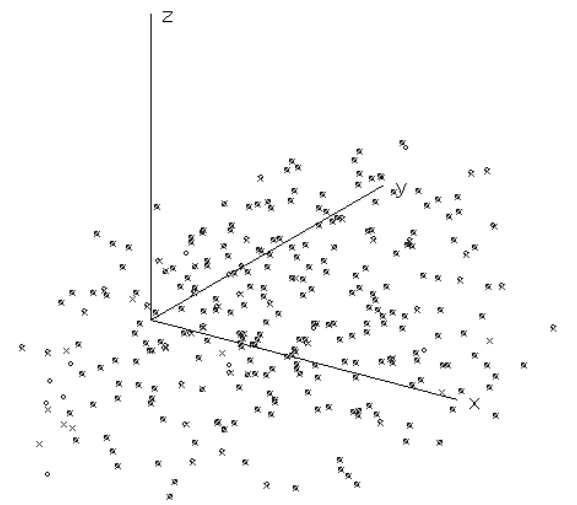

(a) Particle locations for Series \#352/000 (pp)

(Black dot stands for particles detected using GA and Red cross stands for original particles from data sets)

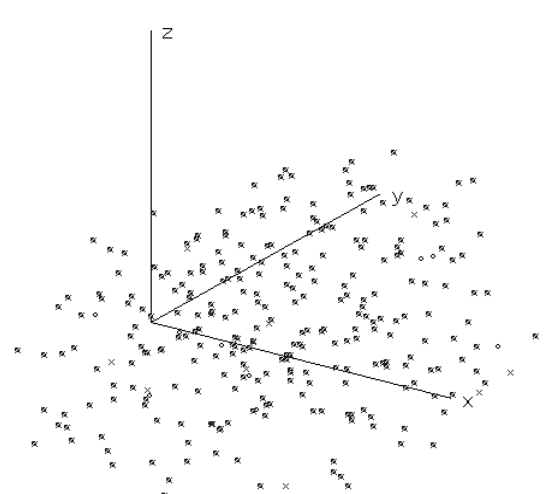

(b) Particle locations for Series \#352/001 (pp)

(Black dot stands for particles detected using GA and Red cross stands for original particles from data sets)

Fig. 7: Comparison of 3-D particle locations for image series \#352 


\section{Conclusion}

The genetic algorithm was successfully applied to the 3-D stereoscopic particle pairing strategy and was confirmed that the genetic algorithm was an effective strategy for the particle pairing of the 3-D stereoscopic particle tracking velocimetry. The pairing problem is modeled as an optimization problem accompanied by physical constraint conditions so as to satisfy the epipolar geometry of the particles and uniqueness of the match pairs. The test results using synthetic particle images (the 3-D PIV Standard Images) showed an increment in the number of correct particle pairs between the two stereoscopic image frames, if compared to those of the conventional method based on a simple nearest-neighbor epipolar line analysis. On the basis of these facts, the performance of the proposed GA algorithm approach is better than the nearestneighbor epipolar line analysis based conventional particle pairing methods for steoroscopic 3-D PTV.

\section{References}

[1] Adrian RJ (2005), Twenty years of particle image velocimetry, Experiments in Fluids 39 : 159169.

[2] Bacakoglu H and Kamel MS (1997), A three-step camera calibration method, IEEE Transactions on Instrumentation and Measurement 46 : 1165-1172.

[3] Grant I and Pan X (1977), The use of neural techniques in PIV and PTV, Measurement Science \& Technology 8 : 1399-1405.

[4] Grant X, Pan X, Romano F and Wang X (1998), Neural-network method applied to the stereo image correspondence problem in three-component particle image velocimetry, Applied Optics 37 : 3656-3663.

[5] Hopfield JJ (1982), Neural networks and physical systems with emergent collective computational abilities, in: Proc. Nat'l Acad. Sci. USA 97 : 2554-2558.

[6] Kent JC (1994), Three-dimensional particle tracking velocimetry: A review, in: Proceedings of 4th FLUCOME symposium, France, pp. 309-314.

[7] Kobayashi T, Saga T and Segawa S (1989), Multipoint velocity measurement for unsteady flow field by digital image processing, Flow Visualization V, Hemisphere 197-202.

[8] Mass HG, Gruen A and Papantoniou D (1993), Particle tracking velocimetry in three dimensional flows, Experiments in Fluids 15 : 33-146.

[9] Murai Y, Oishi Y, Tasaka Y and Takeda Y (2008), Particle tracking velocimetry applied for fireworks: a demonstration of vector field measurement in hundreds meter space, Journal of Visualization 11(1) : 63-70.

[10] Nishino K, Kasagi N and Hirata M (1989), Three-dimensional particle tracking velocimetry based on automated digital image processing, Journal of Fluids Engineering-Transactions of the ASME 11(1) : 384-391.

[11] Ohmi K (2002), Hopfield neural network based particle tracking velocimetry, Journal of Visualization Society of Japan 22(1) : 209-212. 
26 Stereoscopic Correspondence of Particles for 3-Dimensional Particle Tracking Velocimetry by using...

[12] Ohmi K (2003), 3-D particle tracking velocimetry using a SOM neural network, in: Proceedings of 5th International Symposium on Particle Image Velocimetry, \#3112.

[13] Ohmi K (2004), Hopfield neural network based particle tracking velocimetry, in: Proceedings of Fifteenth International Symposium on Transport Phenomena, Bangkok, Thailand, \#20.

[14] Ohmi K (2008), SOM-Based Particle Matching Algorithm for 3-D Particle Tracking Velocimetry, Applied Mathematics and Computation 205 : 890-898.

[15] Ohmi K and Li H (2000), Particle tracking velocimetry with new algorithms, Measurement Science and Technology 11(6) : 603-616.

[16] Ohmi K and Panday SP (2009), Particle tracking velocimetry using the genetic algorithm, Journal of Visualization 12(3) : 217-232.

[17] Ohmi K and Sapkota A (2006), Particle tracking velocimetry using cellular neural network, in: Proceedings of the IEEE World Congress on Computational Intelligence, Vancouver, Canada.

[18] Okamoto K, Nishio S, Kobayashi T, Saga T and Takehara K (2000), Evaluation of the 3D-PIV standard images (PIV-STD Project), Journal of Visualization 3(2) : 115-124.

[19] Okamoto K, Nishio S, Saga T and Kobayashi T (2001), Standard images for particle image velocimetry, Measurement Science and Technology $11: 685-691$.

[20] Ponchaut NF, Mouton CA, Hornung HG and Dabiri D (2005), 3D Particle tracking velocimetry method: Advances and error analysis. In: Galcit Report, California Institute of Technology.

[21] Prasad A and Jensen K (1995), Scheimpflug stereo camera for particle image velocimetry in liquid flows, Applied Optics 34 : 7092-7099.

[22] Raffel M, Willert CE and Kompenhans J, Particle image velocimetry, Springer, Germany.

[23] Salvi J, Armangue X and Batlle J (2002), A comparative review of camera calibrating methods with accuracy evaluation, Pattern recognition 35 : 1617-1635.

[24] Zhang W, Kang JH and Lee SJ (2007), Visualization of saltating sand particle movement near a flat ground surface, Journal of Visualization 10(1) : 39-46. 